\title{
Data assimilation in a multi-scale model
}

\section{Article}

Published Version

Creative Commons: Attribution-Noncommercial-No Derivative Works 4.0

Open Access

Hu, G. and Franzke, C. L. E. (2017) Data assimilation in a multi-scale model. Mathematics of Climate and Weather Forecasting, 3 (1). pp. 118-139. ISSN 2353-6438 doi: https://doi.org/10.1515/mcwf-2017-0006 Available at https://centaur.reading.ac.uk/89879/

It is advisable to refer to the publisher's version if you intend to cite from the work. See Guidance on citing.

To link to this article DOI: http://dx.doi.org/10.1515/mcwf-2017-0006

Publisher: De Gruyter

All outputs in CentAUR are protected by Intellectual Property Rights law, including copyright law. Copyright and IPR is retained by the creators or other copyright holders. Terms and conditions for use of this material are defined in the End User Agreement.

\section{www.reading.ac.uk/centaur}

\section{CentAUR}

Central Archive at the University of Reading

Reading's research outputs online 


\title{
Guannan $\mathrm{Hu}^{*}$ and Christian L. E. Franzke Data Assimilation in a Multi-Scale Model
}

https://doi.org/10.1515/mcwf-2017-0006

Received June 28, 2017; accepted December 23, 2017

\begin{abstract}
Data assimilation for multi-scale models is an important contemporary research topic. Especially the role of unresolved scales and model error in data assimilation needs to be systematically addressed. Here we examine these issues using the Ensemble Kalman filter (EnKF) with the two-level Lorenz-96 model as a conceptual prototype model of the multi-scale climate system. We use stochastic parameterization schemes to mitigate the model errors from the unresolved scales. Our results indicate that a third-order autoregressive process performs better than a first-order autoregressive process in the stochastic parameterization schemes, especially for the system with a large time-scale separation. Model errors can also arise from imprecise model parameters. We find that the accuracy of the analysis (an optimal estimate of a model state) is linearly correlated to the forcing error in the Lorenz-96 model. Furthermore, we propose novel observation strategies to deal with the fact that the dimension of the observations is much smaller than the model states. We also propose a new analog method to increase the size of the ensemble when its size is too small.
\end{abstract}

\section{Introduction}

Forecasting the state of the atmosphere, ocean or climate system requires a numerical model that computes the time evolution of the system, on the one hand, and an estimate for the current state which is used to initialize the model, on the other hand. While the number of observations of the atmosphere and ocean are ever more increasing, we currently still observe the states of the atmosphere and ocean only partially [36].

The uncertainty of predictions is mainly created by two factors: model error and the uncertainty in the initial conditions. Model error is the imperfect representation of the actual system dynamics in a model, which comes from various sources, such as: incomplete dynamics in the numerical model, imprecise knowledge of model parameters in the governing equations, unresolved small-scale processes and numerical approximations, among others [29]. These drawbacks of the model cannot be eliminated because of limited intellectual and computational resources; all discrepancies between a numerical model and the actual system are not necessarily known. The uncertainty in the initial conditions is an additional factor preventing us from achieving skillful forecasts. With advanced techniques, the state of a system can be measured with high precision. But in real world applications, some direct measurements of a system state are not feasible and the observations typically have a much lower resolution in space and time compared to the numerical models. Therefore, the observations are not sufficient to initialize the numerical models and we need to use all useful available observations to estimate initial values of all model variables. Data assimilation is such a method which extracts information from observations and model forecasts and provides improved state estimates of relevant variables and reconstructs the 3-dimensional state variables [13]. Data assimilation is widely applied to atmospheric and oceanic systems $[13,25,36]$ and also extended to the coupled climate system for seasonal forecasts.

\footnotetext{
^Corresponding Author: Guannan Hu: School of Integrated Climate System Science, University of Hamburg, Hamburg, Germany

and Meteorological Institute, University of Hamburg, Hamburg, Germany and Center for Earth System Research and Sustainability, University of Hamburg, Hamburg, Germany, E-mail: guannan.hu@studium.uni-hamburg.de

Christian L. E. Franzke: Meteorological Institute, University of Hamburg, Hamburg, Germany and Center for Earth System Research and Sustainability, University of Hamburg, Hamburg, Germany
}

Oopen Access. (c) BY-NC-ND 02017 Guannan Hu and Christian L. E. Franzke, published by De Gruyter Open. This work is licensed under the Creative Commons Attribution-Non-Commercial-NoDerivs 4.0 License. 
One efficient data assimilation scheme is the ensemble Kalman filter (EnKF) proposed in [17]. It has been applied in a number of different contexts [19], and its skill is examined with the applications to various models, from ocean models $[14,30,38]$ to atmospheric models $[46,60]$, from conceptual climate models [18] to global general circulation models [52]. The performance of the EnKF can be limited by an insufficient ensemble size and sparse observations. A small number of the ensemble members can introduce sampling errors and potentially the forecast error covariance is incorrectly estimated. Covariance inflation and localization are two common methods which are used to correct the error covariance [2, 3, 24, 28, 31]. Grooms et al. [27] pointed out that stochastic subgrid-scale parameterizations have the same effect as the covariance inflation techniques, because they increase the ensemble spread. Moreover, instead of using the ensemble evolved from the past analyzed ensemble states, Tardif et al. [58] used the ensemble formed by randomly drawing model states from preexisting integrations to estimate the background error covariance.

An important problem in contemporary climate science is the assimilation of observations into operational coupled seasonal and decadal prediction models. The climate system can be seen, to first order, as a system with two time scales: the slow ocean and the fast atmosphere. The two-level Lorenz-96 model proposed in [42] is an ideal testbed for numerical experiments considering the computational requirements, possibility for defining the truth and the chaotic, strongly nonlinear nature [18, 44, 45]. Hence, the results can potentially be seamlessly extended to realistic applications with sophisticated and comprehensive models $[8,45]$. The Lorenz-96 system contains coupled equations in two sets of variables. By appropriately choosing the parameter values, we can set the time-scale separation between the two sets of variables and test the sensitivity of data assimilation on different time-scale separations. A major challenge in data assimilation is the presence of model error [29]. To address this issue, we define two kinds of imperfect models in our numerical experiments. They contain the model errors from the imprecise forcing values and unresolved processes, respectively. Instead of resolving small-scale variables in the Lorenz-96 model, we use stochastic parameterization schemes to represent the influence of the unresolved processes on the resolved large-scale variables. The stochastic parameterization schemes are able to mitigate the model errors due to unresolved scales [5, 29, 50, 53, 54]. A perfect model is used to compare with the imperfect models. The perfect model has a specified forcing value which is considered as the true value and resolves the small-scale variables.

The outline of the paper is as follows: In the next section, the two-level Lorenz-96 model is discussed. We will discuss the time-scale separations, imprecise forcing values and stochastic parameterization schemes for the unresolved small-scale variables. In Sec. 3, brief introductions of data assimilation and the EnKF algorithm are presented. We carry out numerical experiments of data assimilation and give the results in Sec. 4. Finally, we end the paper with a discussion and draw a conclusion from our results.

\section{The Lorenz-96 System}

We reformulate the two-level Lorenz-96 model $[42,45]$ in such a way that it explicitly contains a parameter $\varepsilon$ determining the time-scale separation between the two sets of variables of the coupled equations [11, 20]:

$$
\begin{aligned}
\frac{d X_{k}}{d t} & =-X_{k-1}\left(X_{k-2}-X_{k+1}\right)-X_{k}+F-\frac{h}{J} \sum_{j=1}^{J} Y_{j, k}, \\
\frac{d Y_{j, k}}{d t} & =\frac{1}{\varepsilon}\left(-Y_{j+1, k}\left(Y_{j+2, k}-Y_{j-1, k}\right)-Y_{j, k}+h X_{k}\right) .
\end{aligned}
$$

The large-scale $X_{k}$ variables and small-scale $Y_{j, k}$ variables are defined for $k=1, \ldots, K$ and $j=1, \ldots, J$. Each large-scale variable contains $J$ subgrid-scale variables. In our computations, we set the parameters as follows: $K=18, J=20$, coupling coefficient $h=1.0$, and forcing $F=10$ as standard values. The parameter $\varepsilon$ determines the time-scale separation between the $X_{k}$ and $Y_{j, k}$ variables. For $\varepsilon=1.0$, the $X_{k}$ and $Y_{j, k}$ variables have the same time scale. For $\varepsilon<1.0$, the $X_{k}$ variables have a larger time scale than the $Y_{j, k}$ variables. The smaller the value of $\varepsilon$, the larger the time-scale separation. We can also describe the $X_{k}$ variables as slow variables and the $Y_{j, k}$ variables as fast variables when $\varepsilon<1.0$. The model also has a hidden slow time scale 
term [20]: the sum over the fast variables in Eq. (1a).

We list the statistical information of the $X_{k}$ and $Y_{j, k}$ variables of the Lorenz-96 model with different timescale separations in Table. 1. All $X_{k}$ and $Y_{j, k}$ variables have identical statistical properties, respectively. The statistics are calculated from a long-term integration of the Lorenz-96 model with a integration time step $d t=0.001$. The values of time-scale separation are chosen as $\varepsilon=0.125, \varepsilon=0.25, \varepsilon=0.5$ and $\varepsilon=1.0$. Generally, a greater variation of the statistics of the $Y_{j, k}$ variables compared to the $X_{k}$ variables are found. As the time-scale separation increases, the maximum and standard deviation of the $Y_{j, k}$ variables become larger, while the minimum and mean decrease. Both $X_{k}$ and $Y_{j, k}$ variables are approximately Gaussian distributed. A change of the time-scale separation does not alter the distribution.

Table 1: The maximums, minimums, means, and standard deviations of the $X_{k}$ and $Y_{j, k}$ variables in the Lorenz-96 model with different time-scale separations $(\varepsilon)$.

\begin{tabular}{|c|c|c|c|c|c|c|c|c|}
\hline \multirow[b]{2}{*}{$\varepsilon$} & \multicolumn{4}{|c|}{$X_{k}$} & \multicolumn{4}{|c|}{$Y_{j, k}$} \\
\hline & Max & Min & Mean & $\mathrm{Sd}$ & Max & Min & Mean & Sd \\
\hline 0.125 & 13.72 & -7.33 & 2.63 & 3.57 & 17.58 & -13.50 & 1.03 & 2.37 \\
\hline 0.25 & 13.59 & -7.25 & 2.53 & 3.51 & 17.50 & -12.22 & 1.04 & 2.35 \\
\hline 0.5 & 13.33 & -7.35 & 2.45 & 3.54 & 16.17 & -11.50 & 1.15 & 2.16 \\
\hline 1.0 & 13.18 & -9.04 & 2.45 & 3.67 & 12.91 & -10.26 & 1.25 & 1.87 \\
\hline
\end{tabular}

\subsection{Imprecise Forcing}

In a dynamical system which is highly sensitive to the initial conditions, small initial perturbations will lead to widely diverging outcomes. Hence, the medium-term behavior of the system is unpredictable, while the long-term behavior will be determined by the attractor. This behavior is known as deterministic chaos.

The behavior of the Lorenz-96 system is largely determined by the forcing $F$; the value of $F$ determines the presence or absence of chaos, or appearance of other behaviors [20, 42-45]. For a very small value of $F$, all trajectories of the Lorenz-96 system will converge to a steady state, and all $X_{k}$ and $Y_{j, k}$ variables will have an identical value. When the value of $F$ becomes somewhat larger, the steady state solution turns into flow regimes where most solutions are periodic or quasi-periodic, but still not chaotic. When the value of $F$ is large enough (the exact value depends on the dimension of the $X_{k}$ variables), chaotic flows emerge. The three different behaviors of the Lorenz-96 system are illustrated in Fig. 1. The time series of the first component of the $X_{k}$ variables are presented after transients. We observe cyclic behaviors on two time scales when $F=2$. We calculate the maximal Lyapunov exponent (MLE) of the system. The numerical estimates of them are $-1.57,0.0002$ and 7.83 for $F=1, F=2$ and $F=10$, respectively. ${ }^{a}$ The MLE describes the predictability of a dynamical system. Generally, a strictly positive MLE indicates exponential instability and is often considered a signature of deterministic chaos, and a strictly negative MLE indicates stability.

\subsection{Unresolved Processes and Parameterizations}

Regarding many practical applications, we are only interested in predictions of atmospheric processes on a particular scale rather than the detailed evolution of quantities at smaller scales. In this situation, small-scale processes are not necessary to be resolved and in practice, some predictions are impractical to be resolved due

a The MLEs are computed using the fortran code written by Dr. Schubert (https://github.com/seschu/lorenz96_fortran). 

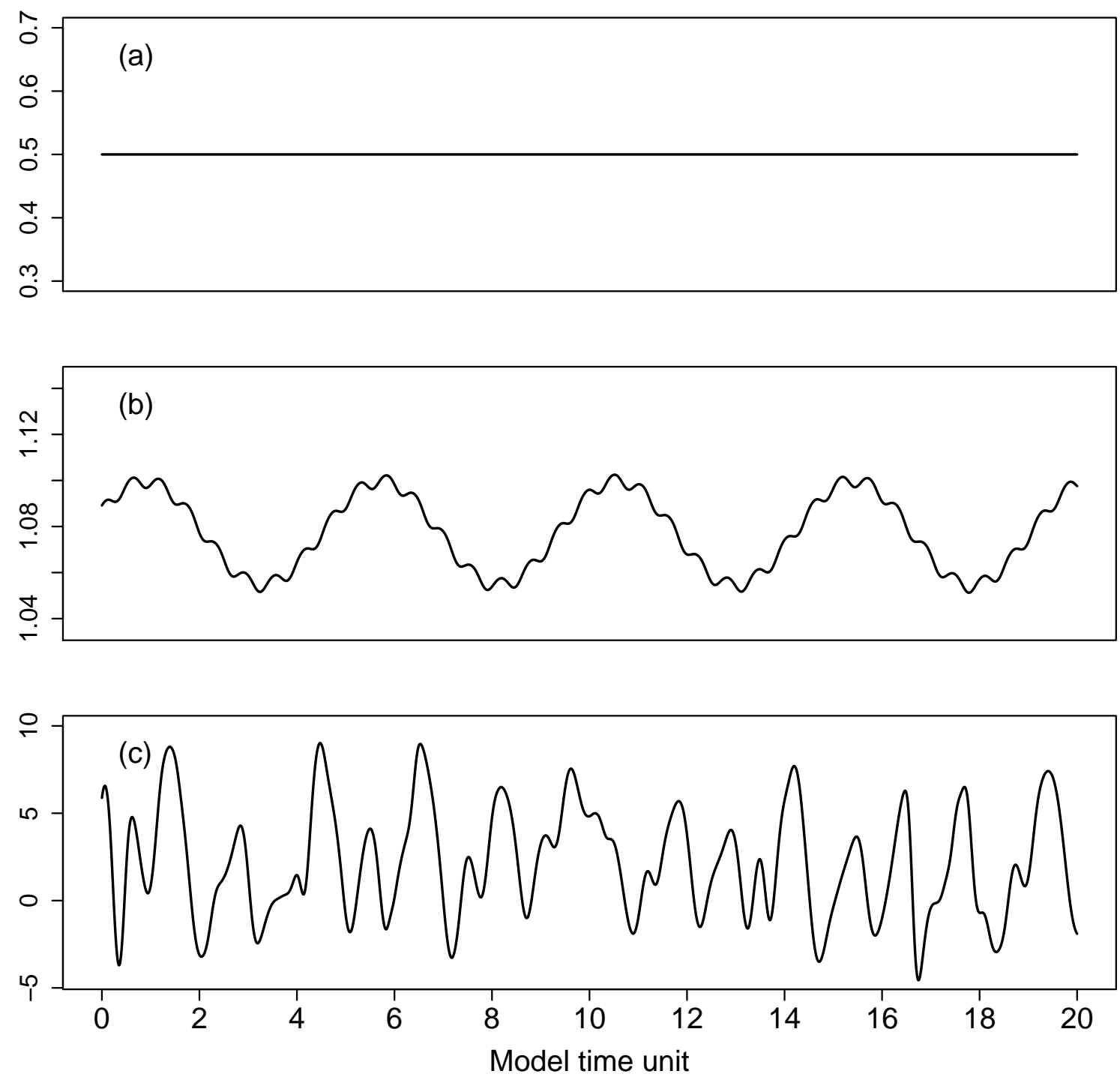

Figure 1: Time series of the $X_{1}$ variable in the Lorenz-96 model with the forcing values (a) $F=1$, (b) $F=2$ and (c) $F=10$. The time-scale separation is $\varepsilon=0.125$.

to computational restrictions. The impacts of these unresolved processes on the evolution of large-scale processes can be represented to some degree by suitable deterministic or stochastic terms [4, 23]. This approach is aimed to obtain a reduced model that involves only the variables of interest. To derive reduced versions of the Lorenz-96 model, we use the parameterization schemes introduced in [65], the key idea in which is to use a polynomial equation and a noise term that represent the model error when only the $X_{k}$ variables are resolved in place of the full dynamics. The polynomial equation with noise is written as follows:

$$
P\left(X_{k}\right)=a_{0}+a_{1} X_{k}+a_{2} X_{k}^{2}+a_{3} X_{k}^{3}+e_{k}(t) .
$$

It is used to replace the last term in Eq. (1a). The $X_{k}$ variables used in Eqs. (2), (5) and (6) are on time step $t$ and for concision, the time index is omitted. Eq. (2) mainly depends on the resolved $X_{k}$ variables and it is based on the notion that the large scales determine the properties of the unresolved subgrid scales [8]. The cubic form of the parameterization is consistent with stochastic climate theory [22, 23, 26, 47-49]. The cubic 
term can be viewed as a nonlinear damping. The coefficients $\left\{a_{0}, a_{1}, a_{2}, a_{3}\right\}$ are determined by fitting a time series of the residuals by a standard least squares method. The time series of the residuals is obtained by taking the difference between the tendency of the full dynamics and reduced tendency with a short time step $\delta t=0.005$ :

$$
P\left(X_{k}(t)\right) \approx\left(\frac{X_{k}(t+\delta t)-X_{k}(t)}{\delta t}\right)-\left(-X_{k-1}(t)\left(X_{k-2}(t)-X_{k+1}(t)\right)-X_{k}(t)+F\right) .
$$

The first four terms of the right-hand side of Eq. (2) are deterministic and the noise term $e_{k}(t)$ is the residual from the polynomial fitting. If we set $e_{k}(t)=0$, then we obtain a deterministic parameterization scheme for the unresolved $Y_{j, k}$ variables. If $e_{k}(t)$ are further fitted by an autoregressive process, then we get a stochastic parameterization scheme. The autoregressive process has the form:

$$
e_{k}(t)=\phi_{1} e_{k}(t-\delta t)+\phi_{2} e_{k}(t-2 \delta t)+\cdots+\phi_{p} e_{k}(t-p \delta t)+\eta(t)
$$

where the parameter $p$ denotes the order of the autoregressive process and determines the memory depth of the time series of the residuals. The $\eta(t)$ are Gaussian noise variables with mean zero and variance $\sigma$. The coefficients $\left\{\phi_{i}, i=1, \cdots, p\right\}$ and the noise variance $\sigma$ are estimated by the Yule-Walker equations [61, 66]. In addition to a first-order autoregressive process, denoted as AR(1) process, which is considered in [65], we also consider an autoregressive process of order 3, denoted as AR(3) process, in our experiments. This is motivated by the fact that the model reduction introduces memory into the reduced system [26] and should be explicitly modeled.

In summary, we have used polynomial equations and autoregressive processes to represent the variability of the unresolved $Y_{j, k}$ variables, and the entire governing equations of two reduced versions of the Lorenz-96 model are given as

$$
\begin{aligned}
\frac{d X_{k}}{d t}= & -X_{k-1}\left(X_{k-2}-X_{k+1}\right)-X_{k}+F-a_{0}-a_{1} X_{k}-a_{2} X_{k}^{2}-a_{3} X_{k}^{3} \\
& -\phi e_{k}(t-\delta t)-\eta(t),
\end{aligned}
$$

and

$$
\begin{aligned}
\frac{d X_{k}}{d t}= & -X_{k-1}\left(X_{k-2}-X_{k+1}\right)-X_{k}+F-a_{0}-a_{1} X_{k}-a_{2} X_{k}^{2}-a_{3} X_{k}^{3} \\
& -\phi_{1} e_{k}(t-\delta t)-\phi_{2} e_{k}(t-2 \delta t)-\phi_{3} e_{k}(t-3 \delta t)-\eta(t),
\end{aligned}
$$

where $d t=\delta t=0.005$. The reduced models contain two parts: the tendency of the $X_{k}$ variables and the stochastic parameterizations of the $Y_{j, k}$ variables. Here, we call the model described by Eq. (5) the L96-AR1 and Eq. (6) the L96-AR3. In comparison to them, we call Eqs. (1a) and (1b) the full dynamic model (FDM).

\begin{tabular}{|c|c|c|c|c|c|c|c|c|c|c|}
\hline \multirow[b]{2}{*}{$\varepsilon$} & \multicolumn{4}{|c|}{ Polynomial terms } & \multicolumn{2}{|c|}{$A R(1)$ process } & \multicolumn{4}{|c|}{$A R(3)$ process } \\
\hline & $a_{0}$ & $a_{1}$ & $a_{2}$ & $a_{3}$ & $\phi$ & $\sigma$ & $\phi_{1}$ & $\phi_{2}$ & $\phi_{3}$ & $\sigma$ \\
\hline 0.125 & 0.18 & 0.42 & -0.002 & -0.0015 & 0.9932 & 0.0031 & 2.73 & -2.58 & 0.85 & $9.6 \mathrm{e}-05$ \\
\hline 0.25 & 0.15 & 0.37 & 0.008 & -0.0019 & 0.9983 & 0.0013 & 2.69 & -2.41 & 0.72 & $1.7 \mathrm{e}-05$ \\
\hline 0.5 & 0.35 & 0.22 & 0.025 & -0.0017 & 0.9994 & 0.0009 & 1.92 & -0.84 & -0.08 & $1.8 \mathrm{e}-05$ \\
\hline 1.0 & 0.72 & 0.09 & 0.022 & -0.0011 & 0.9997 & 0.0005 & 1.73 & -0.47 & -0.26 & $1.4 \mathrm{e}-05$ \\
\hline
\end{tabular}
Parameter values of the L96-AR1 and L96-AR3 are listed in Table. 2. The noise variance $\sigma$ in the L96-AR3 is one or two order of magnitude lower than in the L96-AR1. Note that the estimated parameter values for each $X_{k}$ variable are slightly different. We show the mean values of them in the table and use the corresponding values for each $X_{k}$ variable in the numerical experiments. We compare the probability density functions (PDF) of the

Table 2: Parameter values of the L96-AR1 and L96-AR3.

$X_{k}$ variables in the reduced models to the FDM in Fig. 2. The FDM is integrated with a time step $d t=0.001$ 
and the reduced models are with $d t=0.005$. For all values of the time-scale separation, there are small discrepancies between the FDM and reduced models, and they are smaller when the time-scale separation is larger. Fig. 3 presents the autocorrelation functions (ACF) of the $X_{k}$ variables in the three models. Similar to the comparison of the PDFs, the reduced models well reproduce the ACF of the FDM. Differences are only found in the oscillation of the ACF, especially for the smaller time-scale separations. The L96-AR1 and L96AR3 are able to capture the features of the $X_{k}$ variables in the FDM. The model errors of the reduced models are smaller when the time-scale separation is larger.
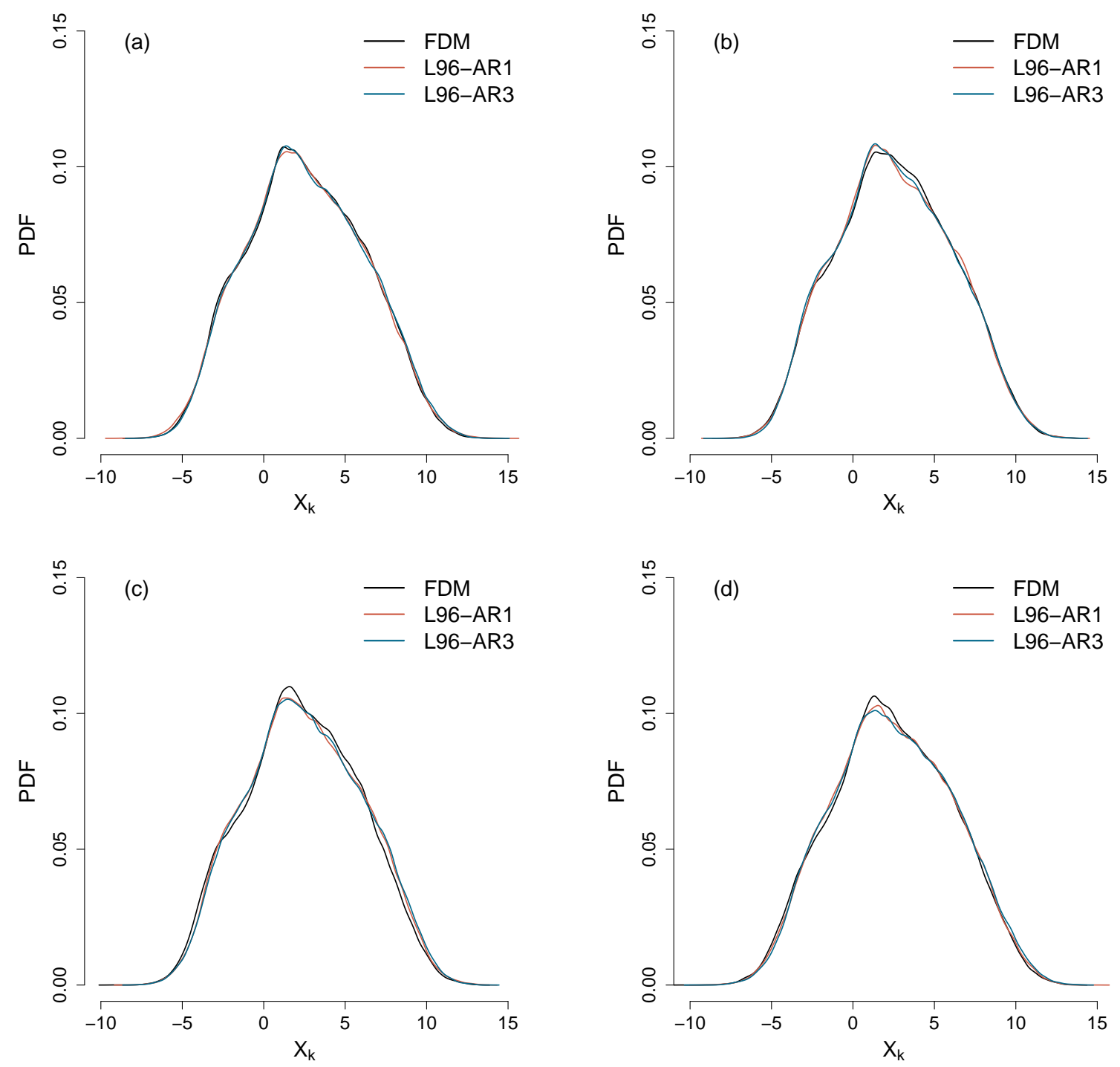

Figure 2: Probability density functions of the $X_{k}$ variables in three models with the time-scale separations (a) $\varepsilon=0.125$, (b) $\varepsilon=0.25$, (c) $\varepsilon=0.5$ and (d) $\varepsilon=1.0$. 

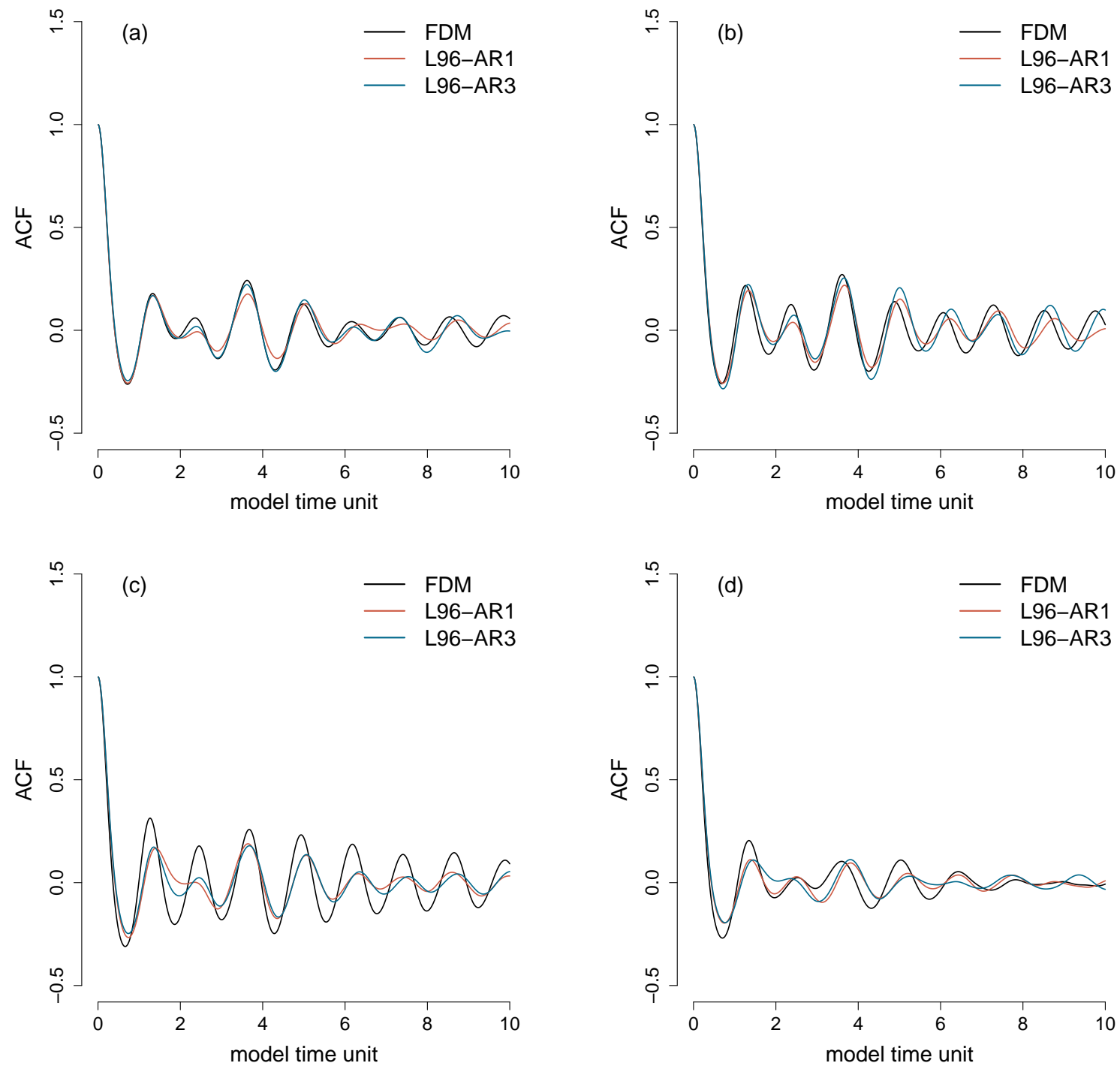

Figure 3: Autocorrelation functions of the $X_{k}$ variables in three models with the time-scale separations (a) $\varepsilon=0.125$, (b) $\varepsilon=$ 0.25 , (c) $\varepsilon=0.5$ and (d) $\varepsilon=1.0$.

\section{Data Assimilation}

Data assimilation is the combination of the information from observational data and a numerical model forecast. Data assimilation schemes can be roughly divided into two categories: ensemble-based methods $[1,17,19,31,32,64]$ and variational methods $[10,39,40,56]$. Variational methods such as three-dimensional or four-dimensional variational data assimilation (3D-Var or 4D-Var) rely on tangent linear operators and adjoint equations. Ensemble-based data assimilation techniques include the ensemble Kalman filter (EnKF), ensemble Kalman smoother (EnKS) and ensemble transform Kalman filter (ETKF), which all depend on statistical estimates from ensemble forecasts. Most practical ensemble-based data assimilation schemes are some kind of approximations of the celebrated Kalman filter [34, 35]. All of them are aimed to either reduce the computing requirements or to improve the statistical forecasts, or both. The EnKF is an efficient ensemblebased data assimilation scheme [16, 17, 19]. This Monte Carlo approximation of the Kalman filter efficiently reduces the computational requirements and directly provides initial perturbations for ensemble forecasts. The main disadvantages of the EnKF are that an insufficient ensemble size and sparse observations limit the 
quality of the produced analysis fields. These shortcomings of the EnKF contrast the advantages of 4D-Var, which produces useful analysis even when observations are sparse and do not need ensembles. The disadvantages of 4D-Var include the developing and maintenance of tangent linear and adjoint models and that many physical parameterization schemes contain step functions. If the assimilation window for 4D-Var is too short, the EnKF performs better than 4D-Var, while for infrequent observations 4D-Var gives more accurate estimates [15, 21, 33, 37, 40, 63]. In practice, hybrid approaches are often adopted [6, 7, 9, 62]. In this paper we use the EnKF.

\subsection{The Ensemble Kalman Filter}

Here, we briefly introduce the algorithm of the EnKF. Comprehensive theoretical aspects and the numerical implementation are provided in [19]. There are two stages involved in a sequential data assimilation methodology: (i) the forecast and (ii) the update stage. During the forecast stage, we use a, possibly nonlinear, model to forecast the system state of dimension $n, \boldsymbol{\psi}_{t}^{f} \in \mathbb{R}^{n}$ and the error covariance of the model forecast, $\boldsymbol{P}_{t}^{f} \in \mathbb{R}^{n \times n}$, where $f$ denotes forecast and $t$ is the time index. When we assimilate observations to the $X_{k}$ variables in the FDM and reduced models, $\boldsymbol{\psi}_{t}^{f}$ represents the $X_{k}$ variables and $n=K$. When we assimilate observations to the $Y_{j, k}$ variables in the FDM, $\boldsymbol{\psi}_{t}^{f}$ represents the $Y_{j, k}$ variables and $n=K J$. In a deterministic model, $\boldsymbol{\psi}_{t}^{f}$ only depends on the former state $\boldsymbol{\psi}_{t-1}^{f}$. $\boldsymbol{P}_{t}^{f}$ is defined in terms of the true state $\boldsymbol{\psi}_{t}^{\text {ref }}$ as:

$$
\boldsymbol{P}_{t}^{f}=\overline{\left(\boldsymbol{\psi}_{t}^{f}-\boldsymbol{\psi}_{t}^{r e f}\right)\left(\boldsymbol{\psi}_{t}^{f}-\boldsymbol{\psi}_{t}^{r e f}\right)^{T}}
$$

where the overbar denotes an expectation value and $T$ means the transpose of a matrix. The true state is unknown and different algorithms are used to estimate $\boldsymbol{P}_{t}^{f}$. For a linear model, the evolution equation is written in discrete form as:

$$
\boldsymbol{\psi}_{t}^{f}=\boldsymbol{F}_{t} \boldsymbol{\psi}_{t-1}^{a}+\boldsymbol{w}_{t},
$$

where $\boldsymbol{F}_{t}$ is a transition matrix, $a$ denotes analysis which is obtained in the update stage, and $\boldsymbol{w}_{t}$ is a Gaussian noise vector with zero mean, representing the model error. The evolution equation of the forecast error covariance becomes

$$
\boldsymbol{P}_{t}^{f}=\boldsymbol{F}_{t} \boldsymbol{P}_{t-1}^{a} \boldsymbol{F}_{t}^{T}+\boldsymbol{Q}_{t},
$$

where $\boldsymbol{Q}_{t}$ is the error covariance matrix for the model error $\boldsymbol{w}_{t}$. With a nonlinear model written as:

$$
\boldsymbol{\psi}_{t}^{f}=\boldsymbol{f}_{t}\left(\boldsymbol{\psi}_{t-1}^{a}\right)+\boldsymbol{w}_{t},
$$

where $\boldsymbol{f}_{t}$ is the forecast operator, the evolution equation of the forecast error covariance is the same as Eq. (9), but with $\boldsymbol{F}_{t}$ being the tangent linear operator of $\boldsymbol{f}_{t}$. In the EnKF, the forecast error covariance is computed by Eq. (7), using the ensemble mean $\overline{\boldsymbol{\psi}}_{t}^{f}$ to replace the unknown true state $\boldsymbol{\psi}_{t}^{\text {ref }}$ [19]. Considering an ensemble of model forecasts with a size of $N$, Eq. (7) becomes

$$
\boldsymbol{P}_{t}^{f}=\frac{1}{N-1} \sum_{i=1}^{N}\left(\boldsymbol{\psi}_{t}^{f, i}-\overline{\boldsymbol{\psi}}_{t}^{f}\right)\left(\boldsymbol{\psi}_{t}^{f, i}-\overline{\boldsymbol{\psi}}_{t}^{f}\right)^{T} .
$$

The second stage in a sequential data assimilation methodology is the update stage, or analysis step. The update stage takes place when the observations $\boldsymbol{d} \in \mathbb{R}^{m}$ of a system state are available. We neglect the time index $t$ of this stage, because all vectors and matrices in this stage are at the same time step. Observations usually have a smaller dimension compared to the model state, $m \leqslant n$. The observation data may also need to be transformed in order to fit the model output. Therefore, a linear measurement operator $\boldsymbol{H} \in \mathbb{R}^{m \times n}$ is used, which relates the true state to the observations

$$
\boldsymbol{d}=\boldsymbol{H} \boldsymbol{\psi}^{r e f}+\boldsymbol{\epsilon},
$$

where $\boldsymbol{\epsilon}$ is measurement errors. $\boldsymbol{H} \in \mathbb{R}^{m \times n}$ maps the model state in $\mathbb{R}^{n}$ to the observation space in $\mathbb{R}^{m}$. Now we have both observations and model forecasts of a system state, and we can use them to estimate the true 
state. The estimated system state is called analysis $\boldsymbol{\psi}^{a}$ in data assimilation. The analysis is determined as a weighted linear combination of forecasts and observations:

$$
\boldsymbol{\psi}^{a}=\boldsymbol{\psi}^{f}+\boldsymbol{K}\left(\boldsymbol{d}-\boldsymbol{H} \boldsymbol{\psi}^{f}\right),
$$

where $\boldsymbol{K} \in \mathbb{R}^{n \times m}$ is called the Kalman gain matrix, which is determined by the error covariance of the forecasts and observations:

$$
\boldsymbol{K}=\boldsymbol{P}^{f} \boldsymbol{H}^{T}\left(\boldsymbol{H} \boldsymbol{P}^{f} \boldsymbol{H}^{T}+\boldsymbol{R}\right)^{-1},
$$

where $\boldsymbol{R} \in \mathbb{R}^{m \times m}$ is the error covariance matrix for the observations and defined as

$$
\boldsymbol{R}=\overline{\boldsymbol{\epsilon} \boldsymbol{\epsilon}^{T}} .
$$

The error covariance of the forecast is also called background error covariance in the update stage. The error covariance of the analysis is obtained by updating the background error covariance:

$$
\boldsymbol{P}^{a}=(\boldsymbol{I}-\boldsymbol{K} \boldsymbol{H}) \boldsymbol{P}^{f} .
$$

Eqs. (13), (14) and (16) are the equations of the standard Kalman filter in the update stage. In the EnKF, the computation of the error covariance of the analysis is not required, because at each analysis step, the background error covariance used in Eq. (14) is directly calculated by Eq. (11) instead of using Eq. (9) to evolve $\boldsymbol{P}^{a}$. In addition, an ensemble of observations is defined as:

$$
\boldsymbol{d}_{i}=\boldsymbol{d}+\epsilon_{i}, i=1, \ldots, N,
$$

where $\epsilon_{i}$ are Gaussian noise variables with zero mean and prescribed variance. The error covariance of the observations becomes

$$
\boldsymbol{R}=\frac{1}{N-1} \sum_{i=1}^{N}\left(\boldsymbol{d}_{i}-\boldsymbol{d}\right)\left(\boldsymbol{d}_{i}-\boldsymbol{d}\right)^{T} .
$$

As described above, the analysis $\boldsymbol{\psi}^{a}$ and the error covariance of the analysis $\boldsymbol{P}^{a}$ are computed during the update stage. They are used to initialize the Eqs. (8) and (9) for linear models and the Eqs. (9) and (10) for nonlinear models in the prediction stage, then the model state and the error covariance are integrated forward in time. Whenever observations are available, the update stage takes place and the analysis and error covariance of the analysis are calculated and used again to initialize the model in the next prediction stage. In the EnKF, the evolution and update of the error covariance of the forecast are not required.

\section{Numerical Data Assimilation Experiments}

In this section, we perform the EnKF with the FDM, L96-AR1 and L96-AR3. We will first describe the experimental settings and the performance measures of the EnKF we use. Next, we will show the influence of the ensemble size on the performance of the EnKF and give a method to inflate an ensemble which has an insufficient size. Then, we will discuss how the distribution of observations affects the performance of the EnKF and give observation strategy. Finally, we compare the imperfect models, in which model errors come from the imprecise parameters and unresolved processes.

\subsection{Experimental Setup}

As described in Sec. 3.1, in order to implement the ensemble Kalman filter (EnKF), a forecast model and observation data are needed. In our numerical experiments, we use the FDM, L96-AR1 and L96-AR3 described in Sec. 2 to generate ensemble forecasts. Observations are created by adding $\boldsymbol{\epsilon} \sim N(0,0.1)$ to the true states 
generated by the FDM with standard forcing value. Our results are not sensitive to the exact value of the observation noise. To generate truth, the FDM is integrated using a fourth-order Runge-Kutta scheme, with a time step of $d t=0.001$. The initial values are randomly sampled from a Gaussian distribution and we take the true trajectory after a transient.

The maximal Lyapunov exponent $\left(\lambda_{\max }\right)$ of the FDM proportionally increases with an increase of the timescale separation. We get $\lambda_{\max } \approx 7.83$ for the time-scale separation $\varepsilon=0.125$. Using the equation given by

$$
E(t)=\frac{E_{0} e^{\lambda_{\max } t}}{1+E_{0}\left(e^{\lambda_{\max } t}-1\right)}
$$

we can estimate the doubling time of small errors in initial conditions [36, 41]. The doubling time is the period of time over which the magnitude of a quantity will double. $E$ represents the root-mean-square average forecast error and it is scaled so that at long forecast leads $E \rightarrow 1$. $E_{0}$ is the initial error and grows to $E(t)$ by time $t$. For a initial error $E_{0}=0.02$ and a $\lambda_{\max }$ of value 7.83 , the doubling time is about 0.09 model time unit (MTU). The value of the initial error is the averaged analysis error of the FDM. In practice, small initial errors in synoptic scales double in about 2 days $[12,57,59]$ and the data assimilation window lengths are $3 \mathrm{~h}, 6 \mathrm{~h}$ or 12h. If we calibrate the interval between two analysis steps in our experiments to the assimilation window in practice via the doubling time, then the interval is too short and the EnKF will diverge. Therefore, we choose the error doubling time 0.09 MTU as the analysis interval.

In our experiments, the analysis and forecast errors are measured in terms of root-mean-square error (RMSE), which is used as the performance measure of the EnKF. The error is defined as the difference between the analysis (forecast) and true state of the control simulation. The equation for calculating the RMSE is given as

$$
R M S E=\sqrt{\frac{1}{K N} \sum_{i=1}^{N} \sum_{k=1}^{K}\left(X_{k}^{a(f), i}-X_{k}^{c t r l}\right)^{2}},
$$

where $k$ counts for each state variable and $i$ for each ensemble member. We only calculate the RMSE of the $X_{k}$ variables in order to make fair comparison between the FDM and reduced models. Unlike the control simulation (generation of the truth), model forecasts are produced by using a fourth-order Runge-Kutta scheme, with a time step $d t=0.005$ (MTU). We implement the EnKF every 18 time steps (analysis interval $d t=0.09$ ) in 10-MTU simulations, which means 111 analysis steps in each simulation. The EnKF converges during the first several analysis steps and after it has converged, we generate 10-MTU forecasts from the desired initial conditions provided at the analysis steps. We calculate the RMSE of the $X_{k}$ variables at each analysis step and each integration time step in forecasts. We use plots of the mean and standard deviation and box plots to present the values of the RMSE of the analysis. We average the values of the RMSE over the same integration time steps in forecasts and show the averaged RMSE value as a function of forecast lead time.

We apply the same observation errors and observe the same subset of variables for all three models. In Secs. 4.2, 4.4 and 4.5, we observe all $X_{k}$ variables. In Sec. 4.3, we observe the $X_{k}$ variables, the $X_{k}$ and $Y_{j, k}$ variables and other 4 different subsets of variables. We choose ensemble size $N=100$ for the reduced models and the FDM when only the $X_{k}$ variables are observed. If we also assimilate observations to the $Y_{j, k}$ variables in the FDM, then we need a larger ensemble size, which is $N=2000$, and also a larger analysis interval, which is $d t=0.27$, to prevent the divergence of the EnKF. The figures in Sec. 4 show the results of the time-scale separation $\varepsilon=0.125$, when we do not give the time-scale separation in the captions.

\subsection{Ensemble Size}

When we apply the EnKF, a proper size of the ensemble has to be chosen. On the one hand, the ensemble size has to be large enough in order to reduce the sampling error which causes the inaccurate estimation of the error covariance of the forecasts. This influences the performance of the EnKF. On the other hand, we need to avoid a too large number of ensemble members which requires and takes up a lot of computing resources. The performance of the EnKF reaches a plateau for a certain ensemble size, beyond this ensemble size the 
improvement in the performance is very small. It is possible to use thousands or even more ensemble members for the Lorenz-96 system in our numerical experiments and we could demonstrate this saturation effect. However, this is impossible for seasonal prediction systems, which only use on the order of 10 to 60 ensemble members.

To estimate a proper ensemble size for our numerical experiments, we implement the EnKF in three mod-

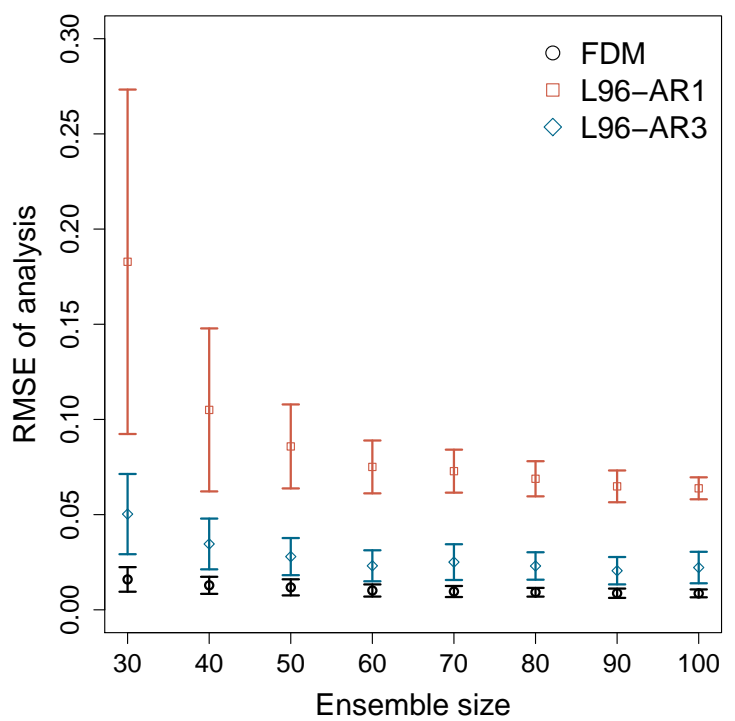

Figure 4: Means and standard deviations of the RMSE of the analysis for different sizes of the ensemble.

els with the ensemble size changing from 30 to 100 with interval 10. Fig. 4 shows the means and standard deviations of RMSE of the analysis for different ensemble sizes. The RMSE of the analysis is larger for the smaller ensembles, especially in the reduced models, and decreases rapidly with an increase in ensemble size, but it cannot drop to zero because of observational noise, imperfection of the model, and the representation of the truth by the ensemble mean. The analysis error of the L96-AR1 reaches to a plateau when the ensemble size is about $N=100$, which is greater than the ensemble sizes the FDM and L96-AR3 need.

Using Monte Carlo simulations to obtain the background error covariance is an efficient modification of the extended Kalman filter (EKF) in the EnKF $[18,51,55]$. It reduces the computational demands in the nonlinear dynamical system (e.g., the computation of the tangent linear operator). However, for large models it also becomes expensive due to the requirement of a sufficient number of ensemble members. An insufficient ensemble size will introduce sampling error and lead to wrong estimates of the background error covariance. We can use some methods to increase the sample size. For instance, we can draw model states from preexisting integrations to form ensembles [58]. However, if these model states are randomly drawn, they will increase the background errors. Therefore, we use observations to find so-called analogs in the model states and use them as additional ensemble members. We define the analogs as the model states which have small values of the root-mean-square deviation to the observations. The values should be smaller than a prescribed threshold. However, if we set the threshold too low, no or only a small amount of analogs can be found and they only slightly improve the performance of the EnKF. If the threshold is too high, the analogs bring too much error which makes the skill of the EnKF worse. To overcome this problem, we only pick the analog which is closest to the observations at each analysis step, and duplicate it many times to form the ensemble with the desired size. For instance, we duplicate the analog 10 times, and use them with 10 regular ensemble members to form a $10+10$-member regular + analog ensemble. The analogs are only used to estimate the background error covariance and not integrated in the prediction stage to make forecasts. We compare a 10-member reg- 
ular ensemble, a 10 + 10-member regular + analog ensemble, and a 20-member regular ensemble in Fig. 5. We only calculate the analysis and forecast errors of the 10 regular ensemble members in the $10+10$-member ensemble. The results show that using analogs to inflate an insufficient ensemble greatly improves the analysis and forecast of the regular ensemble members. Moreover, even for the ensembles with the same size, the EnKF slightly performs better when using analogs to replace part of the regular ensemble members. This is because the analogs have smaller errors than the regular ensemble members in the experiments of the FDM. We may also think of using analogs instead of analysis as the initial conditions. However, the averaged RMSE value of the analogs is greater than the averaged RMSE value of the analysis obtained by using the analogs. In some cases, even though the analogs have larger errors than the regular ensemble members and actually increase the background error, using them to inflate an insufficient ensemble still improves the accuracy of the analysis and forecast. This is found in the experiments of the reduced models (not shown).
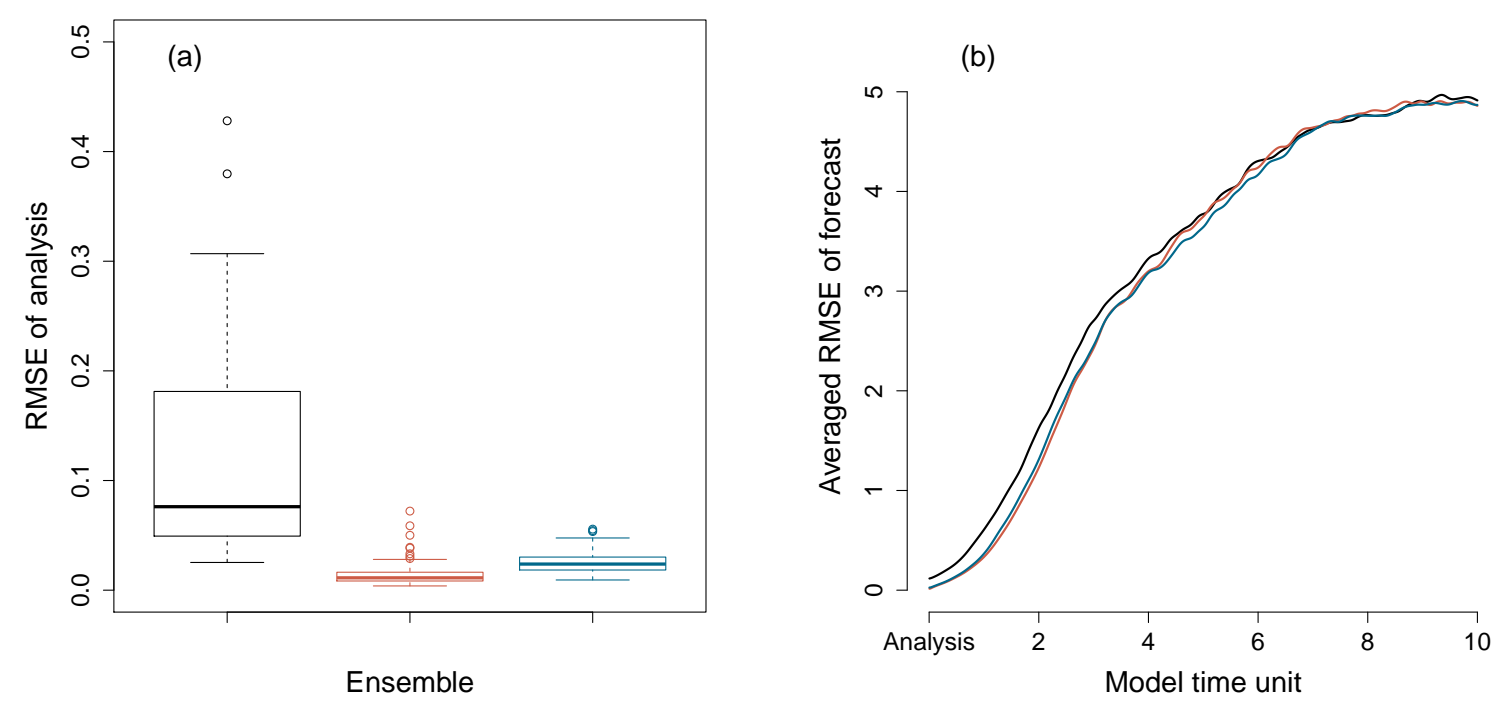

Figure 5: (a) Box plots of the RMSE of the analysis and (b) averaged RMSE of the forecasts as a function of forecast lead time of the FDM. Black: 10-member regular ensemble. Red: 10 + 10-member regular + analog ensemble. Blue: 20-member regular ensemble.

\subsection{Observation Strategy}

In the Lorenz-96 model, the large-scale variables $X_{k}$ are coupled to many small-scale variables $Y_{j, k}$, and they dominate the activity of the $Y_{j, k}$ variables. If the $X_{k}$ variables have large positive values, then the corresponding $Y_{j, k}$ variables, those with the same $k$ values, become active, otherwise they evolve with a small amplitude in time [42]. Unlike this great effect of the $X_{k}$ variables on the $Y_{j, k}$ variables, the impact of the $Y_{j, k}$ variables on the $X_{k}$ variables is much smaller. When we use the reduced models to produce forecasts, we can only assimilate observations to the $X_{k}$ variables, since the $Y_{j, k}$ variables are not resolved any longer. When using the FDM, we also resolve the $Y_{j, k}$ variables. If we need to predict the $Y_{j, k}$ variables, it is necessary that both $X_{k}$ and $Y_{j, k}$ variables are assimilated with observations. If we are only interested in the forecasts of the $X_{k}$ variables, the question arises, whether we should assimilate observations to the $Y_{j, k}$ variables and how large its influence is on the forecasts of the $X_{k}$ variables. There are many more $Y_{j, k}$ than $X_{k}$ variables. Therefore, we would need many more ensemble members to estimate the background error covariance when we assimilate observations to the $Y_{j, k}$ variables. This is time consuming and, thus, undesirable.

Fig. 6 shows the influence of assimilating observations to the $Y_{j, k}$ variables on the analysis and forecasts 
of the $X_{k}$ variables for different values of the time-scale separation. We present the averaged RMSE of the forecasts as a function of forecast lead time, as well as the box plots of the RMSE of the analysis. The $Y_{j, k}$ variables are not modified at the analysis steps when we only assimilate observations to the $X_{k}$ variables. When the time-scale separation is larger, the difference of the forecasts between assimilating and not assimilating observations to the $Y_{j, k}$ variables is smaller. There is a trade-off between the effort of observing the $Y_{j, k}$ variables and assimilating observations to them and the accuracy of the forecasts of the $X_{k}$ variables. Clearly, the effort is independent of the time-scale separation while the improvement of the forecasts becomes less as the time-scale separation increases. This suggests that we can consider assimilating only slow variables in a system with a large time-scale separation.

In our numerical experiments, we can simply generate observations of every variable. But in real world predictions, the dimension of available observations is much smaller than the number of model variables. Therefore, we choose $m=9$, half of the number of the $X_{k}$ variables, as the dimension of the observations for the models. The $X_{k}$ variables can be thought of as values of some atmospheric quantity discretized at $K$ grid points and we can only measure half of them. Imagine that we use Argo floats to measure the temperature and salinity of the ocean and need to consider the changing positions of the Argo floats. For the given observation dimension, there are many different subsets of the $X_{k}$ variables which are observed. Using the equation $C(K, m)=\frac{K !}{(K-m) ! m !}$, we get the number of 9-combinations of 18 which is 48620 . The number of the combinations is too large for us to compare all of them. We consider four cases of observed variables: 1) all $X_{k}$ variables are observed; 2) half of the $X_{k}$ variables with continuous values of $k$ are observed, i.e. $X_{1}, X_{2}, \ldots, X_{9}$; 3) every other of the $X_{k}$ variables are observed, i.e. $X_{1}, X_{3}, \ldots, X_{17}$; and 4) first half $X_{k}$ variables are observed at the first (odd) analysis step, i.e. $X_{1}, X_{2}, \ldots, X_{9}$, then second half $X_{k}$ variables are observed in the next (even) analysis step, i.e. $X_{10}, X_{11}, \ldots, X_{18}$. Fig. 7 presents the box plots of the RMSE of the analysis and averaged RMSE of the forecasts as a function of forecast lead time in these four cases. The results in the three models are consistent: case 3) and 4) show the smallest analysis and forecast errors when only half of the $X_{k}$ variables are observed and the EnKF also converges faster in case 3) and 4) (not shown). In the L96-AR1, the analysis error is greater than the observation error in all three cases of observing half of the $X_{k}$ variables, while this only happens in case 2) of the L96-AR3. We only show the results when the time-scale separation is $\varepsilon=0.125$, because for all values of time-scale separation considered, consistent results are found.

\subsection{Full Dynamic Model with Imprecise Forcing}

The forcing value in our control simulation is $F=10$. Different values of $F$ will result in different dynamics of the system. The larger the difference, the greater the change of the dynamics. A too small forcing value will lead to the appearance of different behaviors of the Lorenz-96 system while a too large forcing value will greatly increase the saturation error, which is the maximal forecast error caused by the uncertainty in the initial conditions. We now choose imprecise forcing values from 9 to 11 with interval 0.2 , which have the forcing error smaller or equal to $10 \%$ of the standard forcing value. We do not want to change the dynamics and reduce the predictive skill of the imprecise models too much. Fig. 8 shows the analysis and background errors of the FDM with different forcing errors. The positive errors mean that the errors are added to the standard forcing value and the negative errors mean subtraction. Although the Lorenz-96 system is chaotic and strongly nonlinear, the RMSE of the analysis linearly increases as the forcing error becomes larger. When the forcing error is greater than 0.6, the analysis error is larger than the observation error. Moreover, the RMSE of the background also has a linear correlation with the forcing error. This is the reason for the linear correlation of the analysis and forcing errors. Because the analysis is obtained by a linear combination of the background and observations (Eq. (13)). There is no obvious difference between the negative and positive errors for the RMSE of the analysis and background. Fig. 9 presents the forecast errors of the FDM with different forcing values as a function of forecast lead time. As the forcing error increases, the forecast error becomes larger. Unlike the analysis error, there is an obvious difference of forecast error between the positive and negative forcing errors: the positive forcing errors lead to faster growths of the forecast errors in the medium-term and long-term predictions and also larger saturation errors. This difference is more obvious when the forcing error 

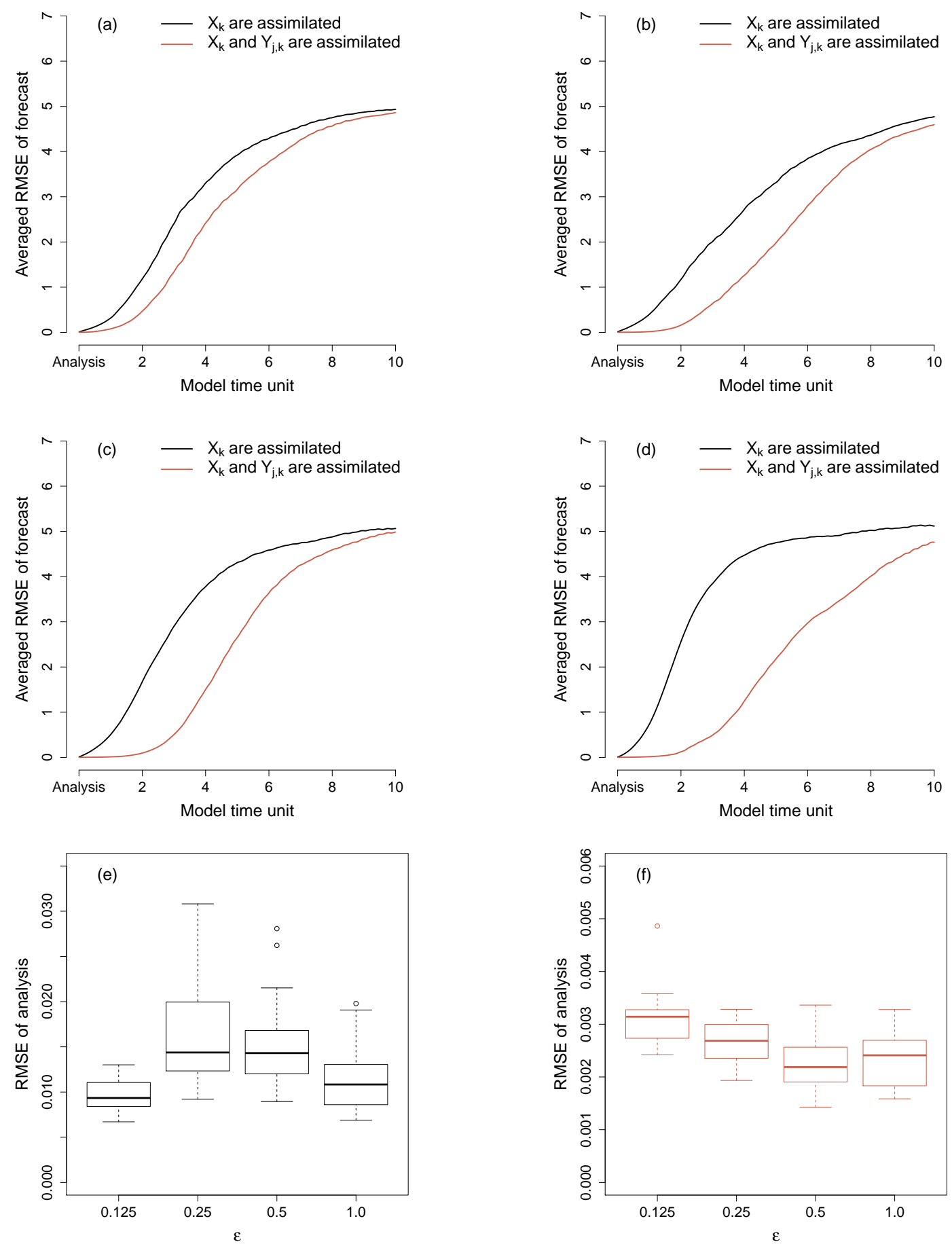

Figure 6: Averaged RMSE of the forecasts as a function of forecast lead time. The time-scale separations are (a) $\varepsilon=0.125$, (b) $\varepsilon=0.25$, (c) $\varepsilon=0.5$ and (d) $\varepsilon=1.0$. Box plots of the RMSE of the analysis: (e) All $X_{k}$ variables and (f) all $X_{k}$ and $Y_{j, k}$ variables are assimilated.

is larger. The reason is that the Lorenz-96 model with a larger forcing value is more chaotic, which is revealed by the larger maximal Lyapunov exponent. We only show the figures for the time-scale separation $\varepsilon=0.125$, and for the other three values of $\varepsilon$, the results are consistent. 

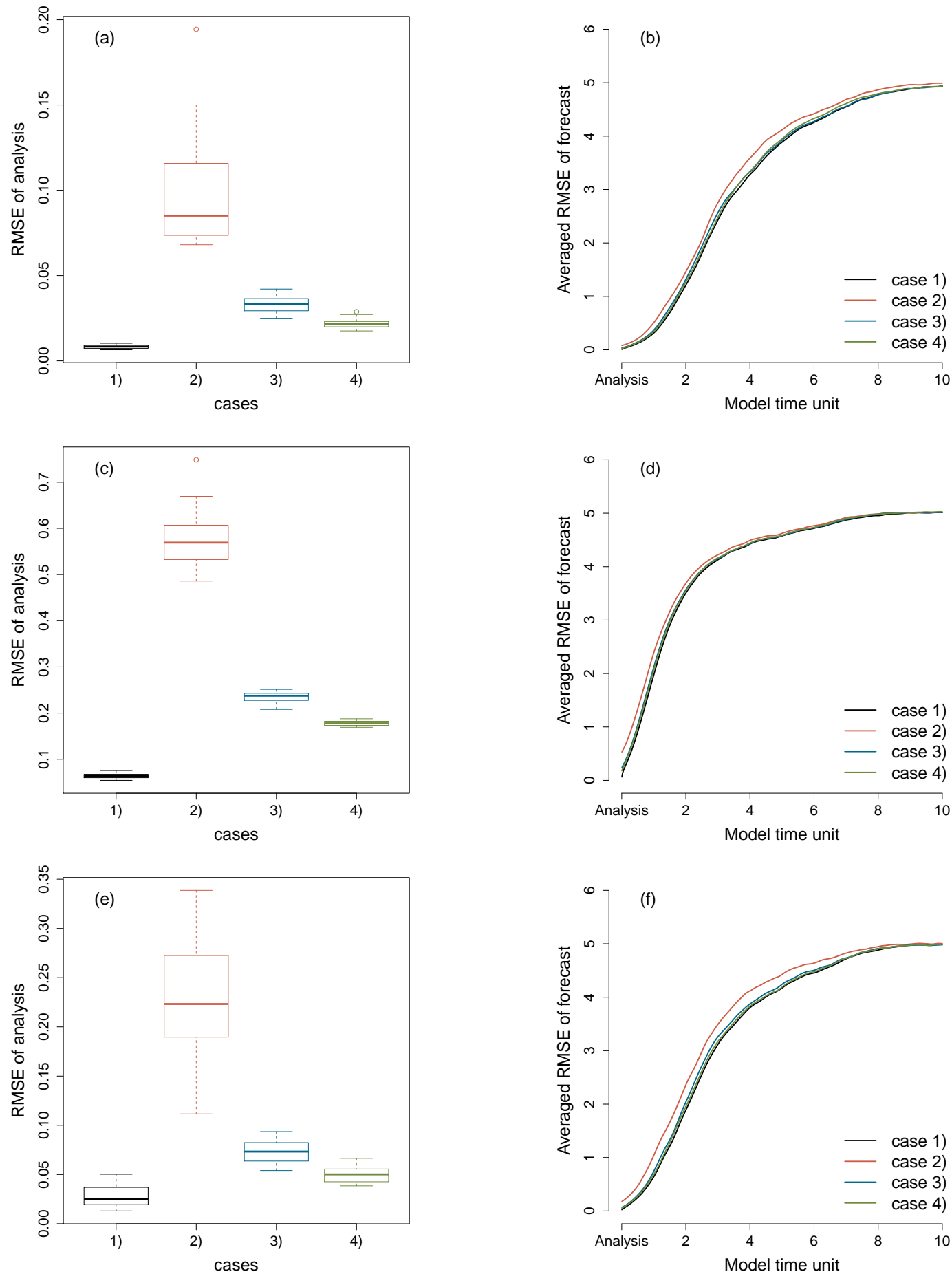

Figure 7: Box plots of the RMSE of the analysis and averaged RMSE of the forecasts as a function of forecast lead time of the FDM (a, b), L96-AR1 (c, d) and L96-AR3 (e, f). Cases 1) - 4) are explained in the main text.

\subsection{Reduced Model with Stochastic Parameterization}

As described in Sec. 2.2, we have defined two reduced models, the L96-AR1 and L96-AR3, which contain stochastic parameterization schemes including a first-oder autoregressive process and an autoregressive process of order 3, respectively. The stochastic parametrization schemes mitigate the model errors arising from 

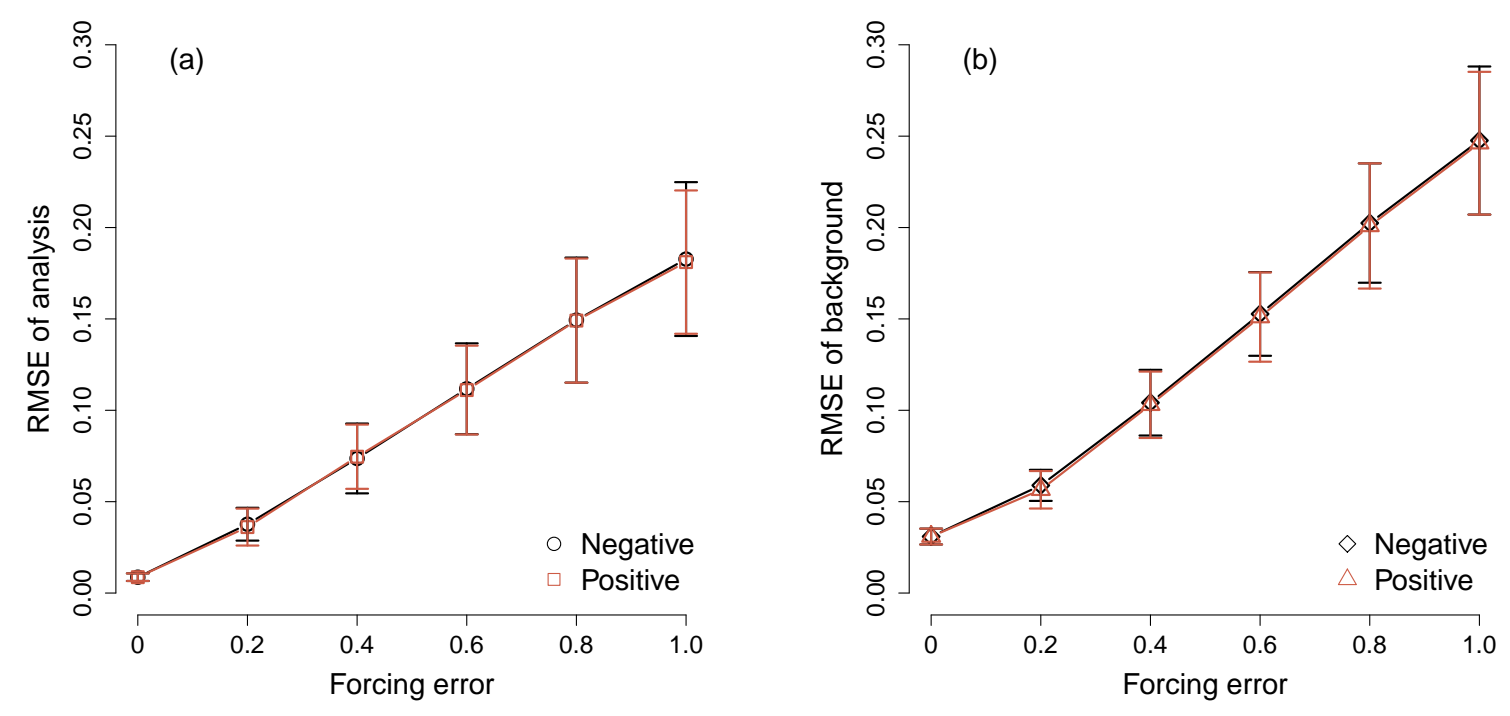

Figure 8: Means and standard deviations of the RMSE of the (a) analysis and (b) background of the FDM with different forcing errors.

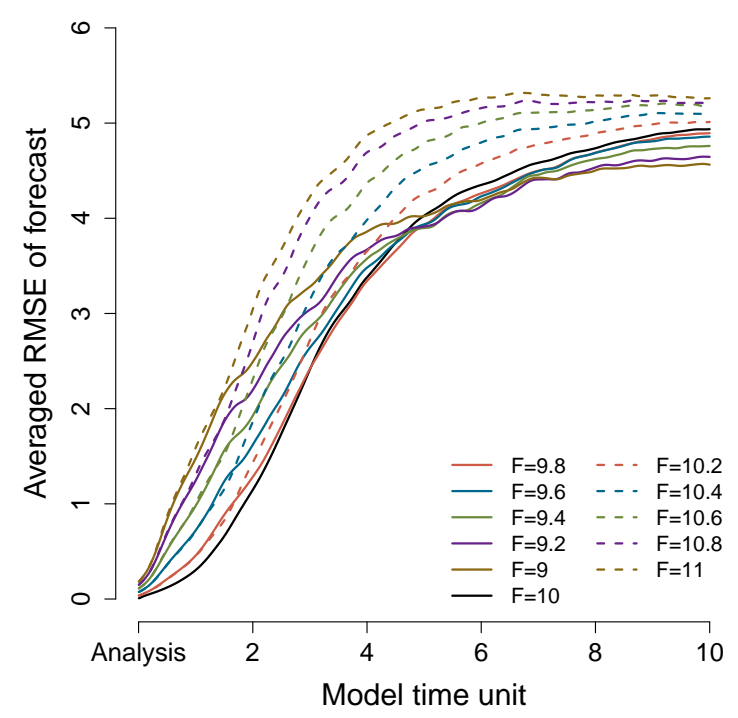

Figure 9: Averaged RMSE of the forecasts of the FDM with different forcing values as a function of forecast lead time.

not resolving the $Y_{j, k}$ variables, but it cannot eliminate the model errors. As shown in Fig. 10, the FDM has the smallest RMSE of the analysis for all values of the time-scale separation considered. For the time-scale separations $\varepsilon=0.125$ and $\varepsilon=0.25$, the L96-AR3 has smaller analysis errors than the L96-AR1. This indicates that the memory is essential for a better representation of the effect of the unresolved scales. We list the means and standard deviations of the ensemble spread and RMSE of the background at analysis steps in Table. 3 . We can find that the accuracy of the analysis mainly depends on the RMSE of the background; the smaller the RMSE of the background, the smaller the RMSE of the analysis. The L96-AR1 has the largest RMSE of the background for all values of the time-scale separation, and it reduces as the time-scale separation decreases. Compared to the L96-AR1, the L96-AR3 has a smaller RMSE of the background, but it increases with a decrease of the time-scale separation. Besides the RMSE of the background, the ensemble spread also influences the 
accuracy of the analysis; the larger the ensemble spread, the smaller the RMSE of the analysis. For time-scale separations $\varepsilon=0.5$ and $\varepsilon=1.0$, even though the L96-AR3 has a slightly smaller RMSE of the background than the L96-AR1, but it has a worse analysis because of the smaller ensemble spread.

Fig. 11 presents the forecast errors of the three models with different time-scale separations. For all values of the time-scale separation, the forecast error grows slower in the L96-AR3 compared to the L96-AR1. The larger the time-scale separation, the better the predictive skill of the L96-AR3. When there is no time-scale separation $(\varepsilon=1.0)$, the forecast errors of the L96-AR1 and L96-AR3 are close. For the time-scale separations $\varepsilon=0.125$ and $\varepsilon=0.25$, the L96-AR3 has the most accurate forecasts which are much better than the L96-AR1.

In summary, the L96-AR3 has a better predictive skill when the time-scale separation is larger. On the
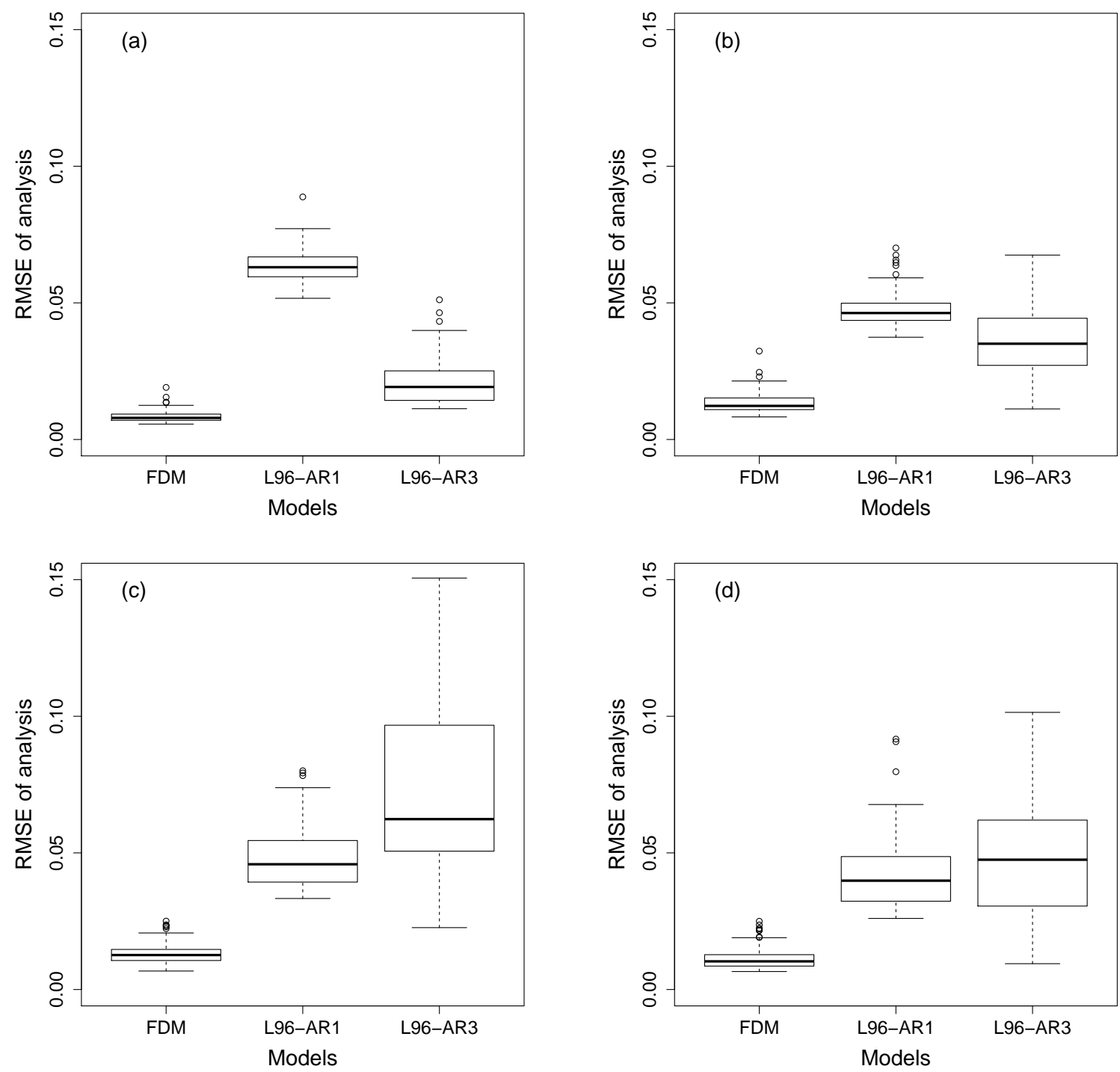

Figure 10: Box plots of the RMSE of the analysis. The time-scale separations are (a) $\varepsilon=0.125$, (b) $\varepsilon=0.25$, (c) $\varepsilon=0.5$ and (d) $\varepsilon=1.0$.

other hand, the short-term predictive skill of the L96-AR1 drops with an increase of the time-scale separation. The L96-AR3 performs better than the L96-AR1, especially for a system with a large time-scale separation. This suggests that memory effects are important for the reduced Lorenz-96 model. 
(a)

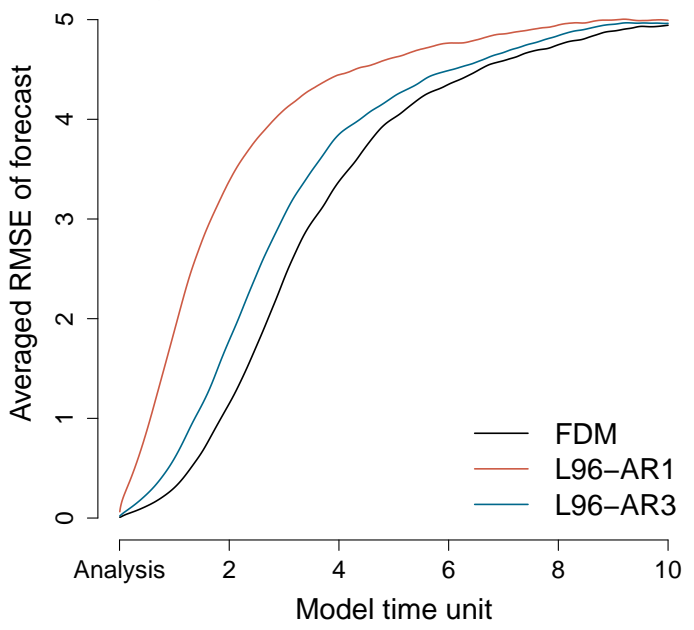

(c)

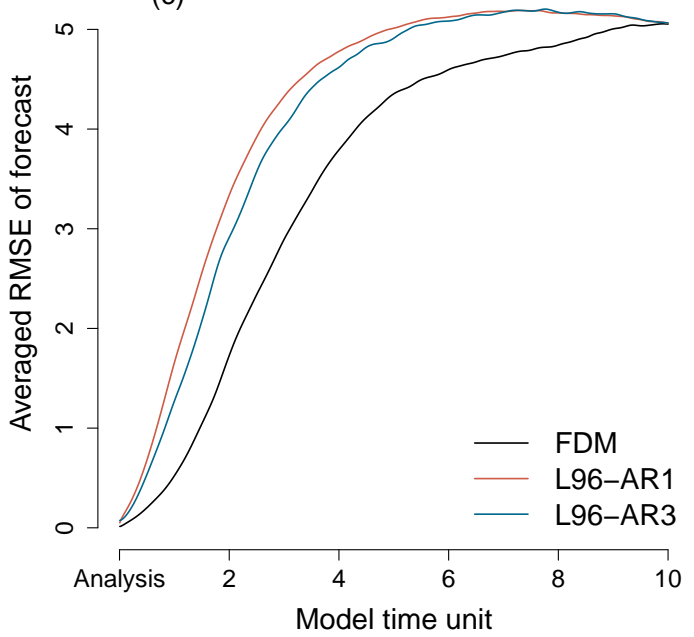

(b)

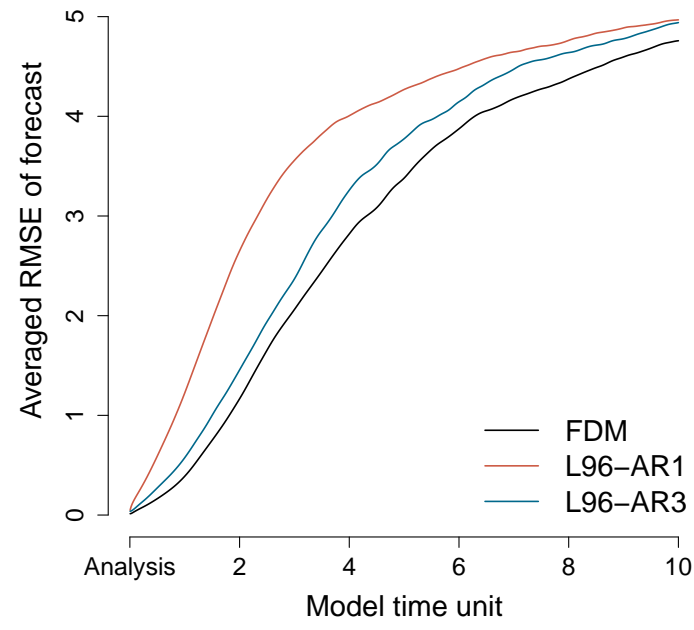

(d)

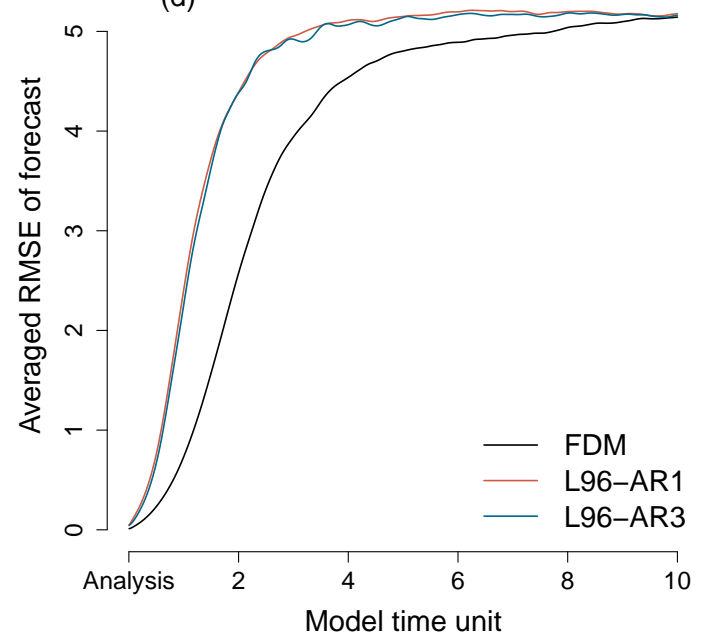

Figure 11: Averaged RMSE of the forecasts as a function of forecast lead time. The time-scale separations are (a) $\varepsilon=0.125$, (b) $\varepsilon=0.25$, (c) $\varepsilon=0.5$ and (d) $\varepsilon=1.0$.

Table 3: The ensemble spread (ES) and RMSE of the background (RMSE_b) of the three models with different time-scale separations $(\varepsilon)$.

\begin{tabular}{|c|c|c|c|c|c|c|}
\hline \multirow[b]{2}{*}{$\varepsilon$} & \multicolumn{2}{|c|}{ FDM } & \multicolumn{2}{|c|}{ L96-AR1 } & \multicolumn{2}{|c|}{ L96-AR3 } \\
\hline & ES & RMSE_b & ES & RMSE_b & ES & RMSE_b \\
\hline 0.125 & $0.02 \pm 0.002$ & $0.03 \pm 0.004$ & $0.24 \pm 0.005$ & $0.25 \pm 0.005$ & $0.04 \pm 0.001$ & $0.06 \pm 0.006$ \\
\hline 0.25 & $0.03 \pm 0.002$ & $0.04 \pm 0.005$ & $0.16 \pm 0.003$ & $0.17 \pm 0.005$ & $0.02 \pm 0.001$ & $0.07 \pm 0.011$ \\
\hline 0.5 & $0.03 \pm 0.001$ & $0.04 \pm 0.005$ & $0.13 \pm 0.003$ & $0.15 \pm 0.007$ & $0.02 \pm 0.001$ & $0.11 \pm 0.032$ \\
\hline 1.0 & $0.02 \pm 0.002$ & $0.03 \pm 0.004$ & $0.10 \pm 0.002$ & $0.13 \pm 0.010$ & $0.02 \pm 0.001$ & $0.10 \pm 0.019$ \\
\hline
\end{tabular}




\section{Discussion and Conclusion}

We have carried out numerical experiments of data assimilation with a prototype multi-scale model of the climate system. We evaluated the effects of different representations of model error and their sensitivity toward time-scale separations. We considered two kinds of model error. The first one is an incorrect parameter setting, by changing the forcing value in the Lorenz-96 model. The forcing value largely determines the behavior of the Lorenz-96 system. The system becomes chaotic only when the forcing value is large enough. For all forcing values considered in our experiments, the Lorenz-96 model is chaotic. The results show that the increase of the forcing error leads to a linear growth of the analysis error, although the Lorenz-96 model is strongly nonlinear. The analysis error is only affected by the absolute value of the constant-in-time forcing error, while the forecast error is also influenced by the sign of the forcing error. For a pair of positive and negative errors which have the same absolute value, the positive errors cause larger forecast errors than the negative errors. The greater the absolute value of the forcing error, the larger the difference in the forecast errors. This is because, if the forcing value is larger, then the maximal Lyapunov exponent of the Lorenz-96 system is also larger. A larger maximal Lyapunov exponent means that the system is more chaotic and unpredictable.

The second type of model error is from unresolved processes. In order to mitigate this kind of model error, we applied stochastic parameterization schemes to the unresolved processes. The stochastic parameterization schemes represent the effects of the unresolved processes on the resolved variables. They contain deterministic and stochastic terms. The deterministic term is a cubic polynomial equation and the stochastic term is an autoregressive process. Our results show that an autoregressive process with a higher order improves over a first-order autoregressive process in parameterizing the fast dynamics in the Lorenz-96 model. The L96-AR3, which contains an autoregressive process of order 3, has a more accurate analysis and a better predictive skill than the L96-AR1, which contains a first-order autoregressive process. This better performance is more obvious when the time-scale separation is larger. Both the L96-AR1 and L96-AR3 more closely reproduces the statistic of the FDM when the time-scale separation is larger, while the short-term predictive skill of the L96-AR1 decreases with an increase of the time-scale separation. Overall, our results on the Lorenz-96 model indicate that the modelling of memory effects improves data assimilation performance.

As discussed in Sec. 3, the sparse observations limit the performance of the EnKF. In realistic circumstances, the dimension of observations is always much lower than the dimension of the model state. Therefore, we want to find out which variables are more useful to be observed for a given dimension of observations. Our results on the Lorenz-96 model indicate that assimilating observations to the fast variables has smaller influence on the forecasts of the slow variables when the time-scale separation between the fast and slow variables is larger. Certainly, we need less observations and a smaller ensemble if we only assimilate observations to the slow variables. Therefore, we can consider not observing the fast variables and not assimilating observations to them for the systems with a large time-scale separation. We also found that the EnKF performs better with the widely distributed observations than with observations concentrated on a region. Moreover, if we observe different subsets of the variables at each analysis step, and make sure all variables are observed in a short observation window, then we can get an accurate analysis which is close to the analysis obtained by observing all variables at one analysis step.

An insufficient size of the ensemble is the other factor which restricts the performance of the EnKF. For large models, a sufficient number of ensemble members is often unaffordable. In the EnKF, the ensembles are used to compute the background error covariance at the analysis steps. To reduce the sampling error caused by a small ensemble, which leads to wrong estimates of the background error covariance, we increase the ensemble size by adding analogs at each analysis step. Our results show that the performance of the EnKF is greatly improved when we add analogs in an ensemble which has an insufficient size. In our experiments, we used a simple method to find the analogs. At each analysis step, we calculated the root-mean-square deviation of each model state to the observations and chose the model state which has the smallest deviation as the analog. Model states were drawn from a preexisting long-term integration. This selection procedure of the analogs can be easily done in the Lorenz-96 model, but in real world prediction system we need a very 
large disk space to store the long-term integrations and the selection procedure is much more time consuming because of the larger resolution and number of the model variables.

Acknowledgement: We thank Prof. V. Lucarini, Dr. F. Lunkeit, and V. M. Galfi for helpful discussions. We are grateful for the support of computing Lyapunov exponents by Dr. S. Schubert. We wish to express our great thanks and sincere gratitudes to Dr. T. Bodai and an anonymous reviewer, who gave us valuable inputs and constructive criticisms. GH is funded by the China Scholarship Council (CSC). CF is supported by the German Research Foundation (DFG) through the collaborative research center TRR 181 "Energy Transfers in Atmosphere and Ocean" at the University of Hamburg and DFG Grant FR3515/3-1.

\section{References}

[1] J. L. Anderson, An Ensemble Adjustment Kalman Filter for Data Assimilation, Monthly Weather Review, 129 (2001), pp. 2884-2903.

[2] - An adaptive covariance inflation error correction algorithms for ensemble filters, Tellus, 59 (2007), pp. $210-224$.

[3] J. L. ANDERSON AND S. ANDERSON, A Monte Carlo implementation of the nonlinear filtering problem to produce ensemble assimilations and forecasts, Mon. Weather Rev., 127 (1999), pp. 2741-2758.

[4] J. Berner, U. Achatz, L. Batte, L. Bengtsson, A. D. L. CÁmara, H. M. Christensen, M. Colangeli, D. R. Coleman, D. CromMELIN, S. I. DolAPTCHIEv, ET AL., Stochastic parameterization: Toward a new view of weather and climate models, Bulletin of the American Meteorological Society, 98 (2017), pp. 565-588.

[5] T. BERRY AND J. HARLIM, Linear theory for filtering nonlinear multiscale systems with model error, Proc. Roy. Soc. London, 470A (2014).

[6] M. BonAVITA, L. ISAKSEN, AND E. HoLM, On the use of EDA background error variances in the ECMWF 4D-Var, Quarterly Journal of the Royal Meteorological Society, 138 (2012), pp. 1540-1559.

[7] M. BONAVITA, L. RAYNAUD, AND L. ISAKSEN, Estimating background-error variances in the ECMWFEnsemble of Data Assimilations system: some effects of ensemble size and day-to-day variability, Quarterly Journal of the Royal Meteorological Society, 137 (2011), pp. 423-434.

[8] H. Christensen, I. MOROZ, AND T. PALMER, Simulating weather regimes: impact of stochastic and perturbed parameter schemes in a simple atmospheric model, Clim Dyn (2015), 44 (2015), pp. 2195-2214.

[9] A. Clayton, A. LORENC, AND D. BARKER, Operational implementation of a hybrid ensemble/4D-Var global data assimilation system at the Met Office, Quarterly Journal of the Royal Meteorological Society, 139 (2013), pp. 1445-1461.

[10] P. CoUrTier, J.-N. THÉPAUT, AND A. HOLlingSWorth, A strategy for operational implementation of 4D-VAR, using an incremental approach, Quarterly Journal of the Royal Meteorological Society, 120 (1994), pp. 1367-1387.

[11] D. Crommelin And E. VAnden-EIjnden, Subgrid-scale parameterization with conditional Markov chains, J. Atmos. Sci., 65 (2008), pp. 2661-2675.

[12] A. DALCHER AND E. KALNAY, Error growth and predictability in operational ECMWF forecasts, Tellus, 39A (1987), pp. $474-491$.

[13] R. DALEY, Atmospheric data assimilation, Journal of the Meteorological Society of Japan, 75 (1997), pp. 319-329.

[14] V. ECHEVIN, P. MEY, AND G. EvENSEN, Horizontal and vertical structure of the representer functions for sea surface measurements in a coastal circulation model, J. Phys. Oceanogr., 30 (2000), pp. 2627-2635.

[15] M. EHRENDORFER AND J. TRIBbia, Optimal Prediction of Forecast Error Covariances through Singular Vectors, J. Atmos. Sci., 54 (1997), pp. 286-313.

[16] G. EvENSEN, Inverse Methods and data assimilation in nonlinear ocean models, Physica (D), 77 (1994a), pp. $108-129$.

[17] - Sequential data assimilation with a nonlinear quasi-geostrophic model using Monte Carlo methods to forecast error statistics, Journal of Geophysical Research, 99 (1994b), pp. 10143-10162.

[18] - Advanced data assimilation for strongly nonlinear dynamics, Monthly Weather Review, 125 (1997), pp. $1342-1354$.

[19] - The Ensemble Kalman Filter: theoretical formulation and practical implementation, Ocean Dynamics, 53 (2003), pp. 343-367.

[20] I. FATKULLIN AND E. VANDEN-EIJNDEN, A computational strategy for multiscale systems with applications to Lorenz 96 model, Journal of Computational Physics, 200 (2004), pp. 605-638.

[21] M. FISHER, M. LEUTBECher, AND G. KELLY, On the equivalence between Kalman smoothing and weak-constraint fourdimensional variational data assimilation, Quarterly Journal of the Royal Meteorological Society, 131 (2005), pp. 3235-3246.

[22] C. L. E. FRANZKE, A. J. MAJDA, AND E. VANDEN-EIJNDEN, Low-order stochastic mode reduction for a realistic barotropic model climate, J. Atmos. Sci., 62 (2005), pp. 1722-1745.

[23] C. L. E. Franzke, T. O'Kane, J. Berner, P. Williams, AND V. LuCARINI, Stochastic climate theory and modelling, WIREs Climate Change, 6 (2015), pp. 63-78. 
[24] G. Gaspari and S. E. Cohn, Construction of correlation functions in two and three dimensions, Quart. J. Roy. Meteor. Soc., 125 (1999), pp. 723-757.

[25] M. Ghil AND P. MALANOTte-Rizzoli, Data assimilation in meteorology and oceanography, Adv. Geophys., 33 (1991), pp. 141266.

[26] G. Gottwald, D. Crommelin, and C. L. E. FranzKe, Stochastic climate theory, in Nonlinear and Stochastic Climate Dynamics, C. L. E. Franzke and T. O’Kane, eds., Cambridge University Press, Cambridge, 2017.

[27] I. Grooms, Y. LEE, AND A. J. MAJDA, Ensemble Filtering and Low-Resolution Model Error: Covariance Inflation, Stochastic Parameterization, and Model Numerics, Mon. Weather Rev., 143 (2015), pp. 3912-3924.

[28] T. M. HAMIL, J. S. WHITAKER, AND C. SNYDER, Distance-dependent filtering of back-ground error covariance estimates in an ensemble Kalman filter, Mon. Weather Rev., 129 (2001).

[29] J. HARLIM, Model Error in Data Assimilation, in Nonlinear and Stochastic Climate Dynamics, C. L. E. Franzke and T. J. O'Kane, eds., Cambridge University Press, Cambridge, 2017, ch. 10, pp. 276-317.

[30] V. Haugen AND G. Evensen, Assimilation of SLA and SST data into an OGCM for the Indian Ocean, Ocean Dynamics, 52 (2002), pp. 133-151.

[31] P. HouteKAmer AND H. Mitchell, Data Assimilation Using an Ensemble Kalman Filter Technique, Monthly Weather Review, 126 (1998), pp. 796-811.

[32] B. Hunt, E. Kostelich, AND I. SzUNYoGH, Efficient data assimilation for spatiotemporal chaos: a local ensemble transform Kalman filter, Physica, 230 (2007), pp. 112-126.

[33] H. JÄRVINEN, E. ANDERSSON, AND F. BOUTTIER, Variational assimilation of time sequences of surface observation with serially correlated errors, Tellus, 51A (1999), pp. 469-488.

[34] R. KALMAN, A new approach to linear filtering and prediction problem, Trans. AMSE J. Basic Eng., 82D (1960), pp. 35-45.

[35] R. KALMAN AND R. BuCY, New results in linear filtering and prediction theory, Trans. AMSE J. Basic Eng., 83D (1961), pp. 95108.

[36] E. Kalnay, Atmospheric Modeling, Data Assimilation and Predictability, Cambridge Univ. Press, 2002.

[37] E. KALNAY, H. LI, T. MiYoshi, S.-C. YANG, AND J. BALlabreRA-POY, 4-D-Var or ensemble Kalman filter?, Tellus, 59A (2007), pp. 758-773.

[38] C. KePPENNE AND M. RieneCKer, Assimilation of temperature into an isopycnal ocean general circulation model using a parallel Ensemble Kalman Filter, J. Mar. Sys., 40-41 (2003), pp. 363-380.

[39] A. LORENC, Analysis methods for numerical weather prediction, Quarterly Journal of the Royal Meteorological Society, 112 (1986), pp. 1177-1194.

[40] - The potential of the ensemble Kalman filter for NWP-a comparison with 4D-Var, Quarterly Journal of the Royal Meteorological Society, 129 (2003), pp. 3183-3203.

[41] E. LoRENZ, Atmospheric predictability experiments with a large numerical model, Tellus, 34 (1982), pp. 505-513.

[42] _ Predictability - a problem partly solved, in In: Proceedings of seminar on predictability, vol. 1, Shinfield Park,Reading, United Kingdom, 4-8 September 1995, In: Proceedings of seminar on predictability, ECMWF, pp. 1-18.

[43] — Designing chaotic models, J. Atmos. Sci., 62 (2005), pp. 1574-1587.

[44] - Regimes in simple systems, J. Atmos. Sci., 63 (2006), pp. 2056-2073.

[45] E. LORENZ AND K. EMANUEL, Optimal sites for supplementary weather observations: simulation with a small model, J. Atmos. Sci., 55 (1998), pp. 399-414.

[46] H. MAdSEn AND R. CAÑIzARes, Comparison of Extended and Ensemble Kalman filters for data assimilation in coastal area modelling, Int. J. Numer. Meth. Fluids, 31 (1999), pp. 961-981.

[47] A. Majda, C. L. E. Franzke, AND D. Crommelin, Normal forms for reduced stochastic climate models, Proc. Natl. Acad. Sci. USA, 106 (2009), pp. 3649-3653.

[48] A. J. MAJDA, C. L. E. FRANZKE, AND B. KHOUIDER, An applied mathematics perspective on stochastic modelling for climate, Phil. Trans. R. Soc. A, 366 (2008), pp. 2429-2455.

[49] A. J. MajdA, I. Timofeyev, AND E. V. EIJnden, Models for stochastic climate prediction, Proc. Nat. Acad. Sci. USA, 96 (1999), pp. 14687-14691.

[50] Z. Meng And F. Zhang, Tests of an Ensemble Kalman Filter for Mesoscale and Regional-Scale Data Assimilation. Part II: Imperfect Model experiments., Mon. Wea. Rev., 135 (2007), pp. 1403-1423.

[51] R. N. Miller, M. Ghil, AND F. GaUthiez, Advanced Data Assimilation in Strongly Nonlinear Dynamical Systems, J. Atmos. Sci., 51 (1994), pp. 1037-1056.

[52] H. Mitchell, P. HOUTEKAMER, AND G. PELLERIN, Ensemble size, and model-error representation in an Ensemble Kalman Filter, Monthly Weather Review, 130 (2002), pp. 2791-2808.

[53] L. Mitchell AND A. CARRASSI, Accounting for model error due to unresolved scales within ensemble Kalman filtering, Quart. J. Roy. Meteor. Soc., 141 (2015), pp. 1417-1428.

[54] T. N. PALMER, A nonlinear dynamical perspective on model error: A proposal for non-local stochastic-dynamic parameterization in weather and climate prediction models, Quart. J. Roy. Meteor. Soc., 127 (2001), pp. 279-304.

[55] D. T. PhAm, Stochastic Methods for Sequential Data Assimilation in Strongly Nonlinear Systems, Mon. Weather Rev., 129 (2001), pp. 1194-1207. 
[56] Y. SASAKI, Numerical variational analysis with weak constraint and application to surface analysis of severe storm gust, Monthly Weather Review, 98 (1970), pp. 899-910.

[57] A. J. Simmons, R. MUREAU, AND T. PETROLIAGis, Error growth estimates of predictability from the ECMWF forecasting system, Quarterly Journal of the Royal Meteorological Society, 121 (1995), pp. 1739-1771.

[58] R. TARDIF, G. HAKIM, AND C. SNYDER, Coupled atmosphere-ocean data assimilation experiments with a low-order model and CMIP5 model data, Clim. Dyn., 45 (2015), pp. 1415-1427.

[59] Z. TотH AND E. KALNAY, Ensemble forecasting at NMC: the generation of perturbations, Bull. Amer. Meteor. Soc., 74 (1993), pp. 2317-2330.

[60] M. vAn Loon, P. BuILTJEs, AND A. SEgERS, Data assimilation of ozone in the atmospheric transport chemistry model LOTUS, Environ. Modelling Software, 15 (2000), pp. 603-609.

[61] G. WALker, On Periodicity in Series of Related Terms, Proceedings of the Royal Society of London, 131 (1931), pp. 518-532.

[62] X. WANG, D. PARRISH, D. KLEIST, AND J. WhITAKER, GSI 3DVar-Based Ensemble-Variational Hybrid Data Assimilation for NCEP Global Forecast System: Single Resolution Experiments, Monthly Weather Review, 141 (2013), pp. 4098-4117.

[63] J. Whitaker, G. Compo, AND J.-N. Thepaut, A Comparison of Variational and Ensemble-Based Data Assimilation Systems for Reanalysis of Sparse Observations, Monthly Weather Review, 137 (2009), pp. 1991-1999.

[64] J. Whitaker AND T. HAmill, Ensemble Data Assimilation without Perturbed Observations, Monthly Weather Review, 130 (2002), pp. 1913-1924.

[65] D. WiLKs, Effects of stochastic parameterizations in the Lorenz '96 model, Quart. J. Roy. Meteor. Soc., 131 (2005), pp. 389407.

[66] G. U. YULE, On a Method of Investigating Periodicities in Disturbed Series, with Special Reference to Wolfer's Sunspot Numbers, Philosophical Transactions of the Royal Society of London, 226 (1927), pp. 267-298. 